\title{
Transistor Avalanche Non-Coherent Pulse Generator
}

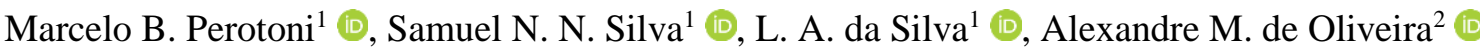 \\ ${ }^{1} U F A B C$, Av. Dos Estados 5001 Santo Andre SP Brazil.mperoconsult@gmail.com, \\ samuel.elektroniker@gmail.com,leandro.aurelio@hotmail.com \\ ${ }^{2}$ IFSP Cubatao, Rua Maria Cristina 50 Cubatao, SP Brazil. amanicoba@ifsp.edu.br
}

\begin{abstract}
A transistor avalanche noise generator (TANG) is described. It is based on an off-the-shelf RF transistor with an integrated planar monopole. From a $70 \mathrm{~V}$ DC power it generates sub-nanosecond pulses with amplitudes close to $1 \mathrm{~V}$. The proposed solution does not employ step recovery diodes, and it is based as the name implies on the operation of a bipolar transistor crossing into the avalanche region. Radiation measurements showed energies close to $-40 \mathrm{dBm}$ at frequencies near $600 \mathrm{MHz}$, with energies reaching the range of $1 \mathrm{GHz}$.
\end{abstract}

Index Terms - Avalanche Breakdown, Pulse Circuits, Pulse Excited Antennas.

\section{INTRODUCTION}

Fast pulse generators have several applications, such as electromagnetic noise generators, medical treatment, Ultra-Wideband (UWB) radars [1], ground penetrating radar (GPR) [2], optical switches [3], etc. A Marx generator based on special avalanche transistors was analyzed in [4], with a total of ten stages and powered with DC voltages from $250 \mathrm{~V}$ up to $284 \mathrm{~V}$. The signal was visualized with a $1.5 \mathrm{GHz}$ oscilloscope on a load resistor. It was not seen the radiated signal since the system had not been connected to a radiant system. A chain of four series-connected power transistor (2N3055) were excited to enter into avalanche mode, generating a $666 \mathrm{~V}$ pulse which is connected to a $100 \mathrm{MHz}$ bow-tie antenna [2]. In [5], a string of 22 avalanche transistors FMMT417 (connected as diodes; emitter and bases short-circuited, breakdown voltage of $270 \mathrm{~V}$ ), is powered up with a $6 \mathrm{kV} \mathrm{DC}$ voltage. A pulse with rise time of $3 \mathrm{~ns}$ and amplitude of $2.5 \mathrm{kV}$ is generated and extracted by means of a coaxial transmission line. The use of avalanche transistors as UWB source is limited due to the repetition rate, which may potentially create thermal damage to the device, so step recovery diodes (SRD) are indicated, though operating at lower amplitudes. In [6] a UWB pulse generator employing SRDs followed by a shape-forming network outputs a monocycle pulse, with amplitude of $25 \mathrm{~V}$ and pulse width of $500 \mathrm{ps}$. A negative point regarding step recovery diodes is their not easy availability, due to the potential dual-use, with restrictions on their commercialization [2]. Another approach to pulse generation to be employed in a carrier-free radar and communication systems uses the LCR concept (Large Current Radiator), a non-resonant radiant system able to withstand high currents with low voltages, as in [7], where it is coupled to an avalanche transistor generating high amplitude fields (4.2 $\mathrm{mA} / \mathrm{m}$ at 5 meters of distance), with pulses with rise times of about $1 \mathrm{~ns}$. LCR antennas present Brazilian Microwave and Optoelectronics Society-SBMO received 28 Nov 2019; for review 5 Dec 2019; accepted 9 Apr 2020 
some pitfalls though, among them their inherent low efficiency [8]. In [9] an avalanche oscillator is implemented generating a high power pulse $(302 \mathrm{~W})$, which had its output optimized to a $50 \Omega$ impedance, connected through a balloon into a TEM horn antenna. Concerning the computer simulation of avalanche circuits, an improved model of a transistor is presented and the parameter named avalanche conductance $g_{\mu}$ is introduced, to better describe gain and stability figures [10].

This article covers a broadband noise generator based on an off-the-shelf bipolar device (applications in RF amplifiers), not specifically avalanche transistor. It is excited with a moderate (70 $\mathrm{V})$ DC voltage and generates pulses which are extracted by means of a monopole planar antenna, whose spectrum covers reaches up to $2 \mathrm{GHz}$. Section II of the article covers the circuit and its working fundamentals; section III the respective measured pulse waveform and section IV the radiated energy, measured with a spectrum analyzer.

\section{CIRCUIT}

Avalanche breakdown occurs when the minority carriers inside a junction cross the depletion region with enough kinetic energy to break covalent bonds inside the other atoms. As a consequence, other nearby carriers are set free. This kinetic energy comes at the expense of an external high-amplitude electric field. The process is called avalanche because the generated carriers set in turn others carriers (electron-hole pairs) free, thereby causing a high amplitude current. In spite of the name, the avalanche breakdown is not destructive as long as power dissipation limits are respected. The process of generation is limited by the external circuitry that controls the pulse repetition frequency and subsequently the average thermal conditions.

The basic circuit for the avalanche noise generator is shown in Fig. 1. The capacitor $C 1$ charges through $R I$ (current path shown in the circuit at top right, interrupted line), with the time constant R1.C1, that should be larger than $1 / f_{T}$ ( $f_{T}$ is the transition frequency of the transistor), so that the device is still able to respond to the pulses properly. After the onset of the avalanche (current path shown in the circuit at bottom right, interrupted line), the capacitor discharges through the semiconductor, which is in the ON state, starting again a new cycle due to the low voltage that is applied to the collector, turning the device OFF. That process is the low frequency (repetition rate) that modulates the fast pulses. Unlike other approaches [2], [3] and [7] this oscillator does not have a trigger pulse, oscillating in a free-running fashion. The capacitor $C 1$ (sometimes replaced by a coaxial transmission line section [12]) controls the repetition rate. The free-running characteristic was chosen in this design in order to achieve a reduced number of components and the respective parasitic elements on the board. $R 2$ limits the maximum current on the avalanche mode, and it was chosen close to the reference impedance $50 \Omega$. $R 3$ was experimentally found; on its lower bound it generates a current too large in the base whereas on its upper limit the voltage drop over it becomes too large, turning the transistor OFF. It was experimentally found to be between $6.8 \mathrm{k} \Omega$ and $10 \mathrm{k} \Omega$.

If the transistor under avalanche (ON condition) generates a short generic pulse $p(t)$, it is further 
subjected to a repetition rate $s(t)$, the latter in first order analysis controlled by the time constant R1.Cl. The final product $f(t)$ can be written as:

$$
f(t)=\sum_{n=1}^{\infty} p(t) \cdot s\left(t-n \cdot t_{s}\right)
$$

where $t_{s}$ is the inverse of the PRF (Pulse repetition frequency). In the frequency domain (angular frequency variable $\omega$ ) it is possible to write $-F, P$ and $S$ are the Fourier Transform of $f, p$ and $s$, respectively:

$$
F(\omega)=P(\omega) * S(\omega)
$$

So the frequency spectrum of the composed signal is the convolution of the individual pulse (ideally with a high frequency content) and the repetition signal $\mathrm{S}(\omega)$ (lower frequency). The periodic pulse than fills the electromagnetic spectrum in a non-deterministic fashion, since the transistor avalanche pulse $p(t)$ varies with time.

The used transistor has a transition frequency $f_{T}$ of $6 \mathrm{GHz}$ - in theory the higher the $f_{T}$ the higher the generated noise frequency generated by the TANG circuit. It has a nominal avalanche voltage (base-collector VCBO) of $20 \mathrm{~V}$. For the experiment it was used a voltage $V 1$ of $70 \mathrm{~V}$, according to the schematic of Fig. 1. The voltage level that feeds the transistor has to be higher than the threshold VCBO in order to trigger the avalanche effect, but its exact value is experimentally defined. It was observed with different transistors that the oscillation occurs at values of $V 1 \cong 2.5$.VCBO.

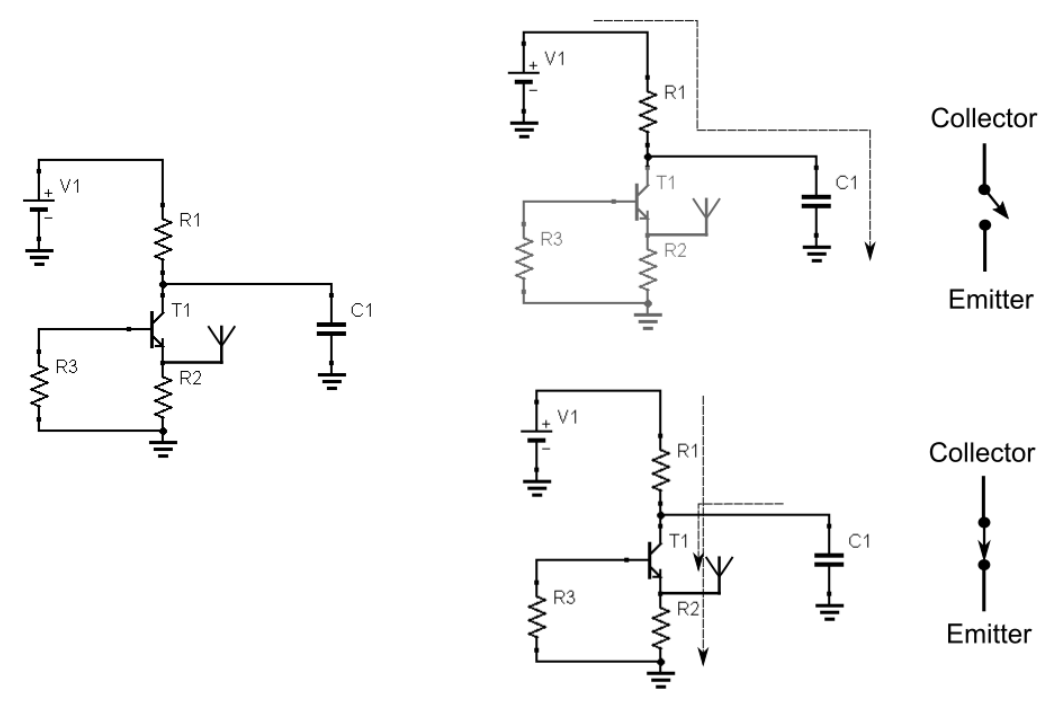

Fig. 1. Basic circuit for the noise generator. $R l=100 \mathrm{k} \Omega, R 2=47 \Omega$ and $R 3=8 \mathrm{k} \Omega$. $C l=2 \mathrm{pF}$ and the transistor is the BRF91A. The collector and emitter are shown in the two states (ON under avalanche and OFF when the capacitor is charging). Current paths shown in interrupted lines.

There are two junctions in the transistor - base-collector (BC) and base-emitter (BE), which may give rise to the respective breakdowns. The breakdown taking place on the $\mathrm{BC}$ junction (unlike the BE counterpart) is not destructive [11], as long as the device thermal limits are not crossed. As Fig. 2 shows, the avalanche area shows a clear negative resistance region, adequate for the onset of 
oscillations. The device is biased by an external circuit so that it goes from point (1), where it is in cutoff, to (2), where the avalanche takes place, with a high-intensity current. A short, high-amplitude current pulse then brings back the circuit to the point (1) again, starting the process over. In the circuit here analyzed the sudden conduction on point (2) discharges the capacitor, bringing back the operation point to point (1).

The existence and waveshape of the oscillations depend on the transistor characteristics. The same transistors but originated from different batches showed irregular behaviors, some units oscillating while others without any instability. The circuits were experimentally tested by slowly increasing the DC voltage until the onset of oscillations is verified on an oscilloscope. That was seen not only with the BRF91 but also with lower frequency bipolar counterparts such as 2N2222 and 2N369A.

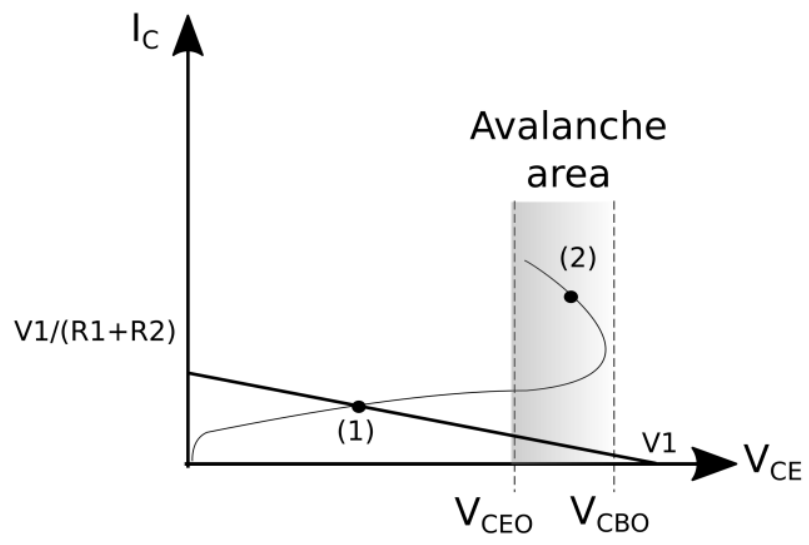

Fig. 2. Load line for the TANG.

Initial tests measured the electromagnetic noise generated with the circuit without any radiant system. The broadband nature of the signal and the difficulty of determining the output impedance on the transistor emitter (it operates on a highly non-linear region) directed the solution to a simple planar monopole antenna, shown in Fig. 3. The antenna was directly connected to the emitter resistor $R 2$ depicted in Fig. 1. The length of the antenna was cut to be a quarter-wavelength at the frequency close to $600 \mathrm{MHz}$, since it was in a part of the spectrum where existing radiation was non-existent. It was made of copper foil, with dimensions of $141.4 \mathrm{~mm}$ length and $20 \mathrm{~mm}$ width. 


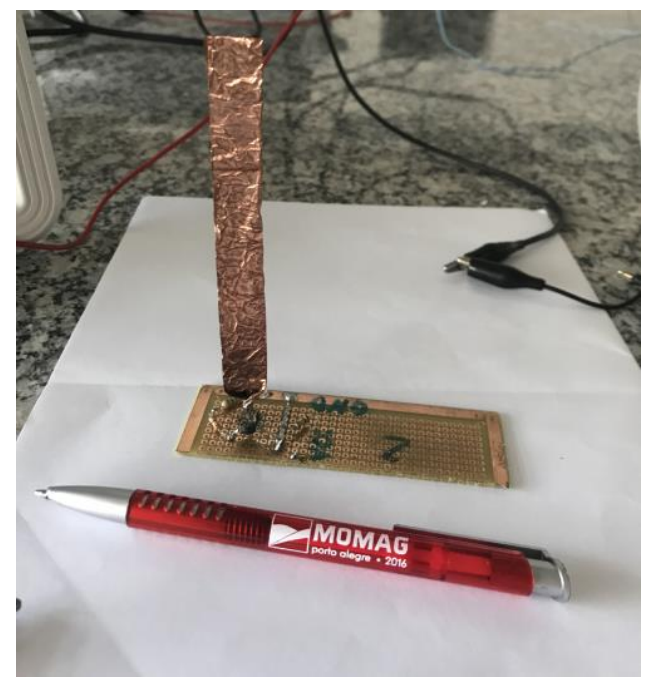

Fig. 3. Circuit with planar monopole antenna.

The TANG circuit aims its use as a jammer cell, to investigate the blocking of ordinary UHF communication systems, particularly with mobile phones and with drones. Its small dimensions enable the use of several TANGS operating in parallel, generating non-coherent EM noise thereby covering with higher efficiency a large frequency spectrum.

Further improvement on the frequency operation can be achieved by the use of SRD devices in series with the avalanche current, so that a multiplier frequency effect can be achieved [13].

\section{PULSE WAVEFORM}

In order to visualize the pulse in the time domain an $8 \mathrm{GHz}$ Keysight Infiniium MSO804A oscilloscope was employed. In Fig. 4 two curves are shown, one captured with a high impedance (1 $\mathrm{M} \Omega$ ) probe and other with $50 \Omega$ direct connection. The high impedance probe had a maximum frequency of $500 \mathrm{MHz}$ whereas the direct connection can, in theory, achieve the instrument maximum frequency. It can be seen from the curves that the direct connection results in a sampled waveform with a smaller amplitude, due to the loading applied on the TANG and also impedance reflections, though with a better fidelity on the sampled fast transitions.

From Fig. 4 it is possible to measure the rise time to be about $0.31 \mathrm{~ns}$ (for the $50 \Omega$ input) and $1.7 \mathrm{~ns}$ (high impedance input). The normalized power spectrum of both pulses is shown in Fig. 5. The higher upper limit frequency of the direct $50 \Omega$ connection captures a higher bandwidth, though with a smaller signal to noise ratio. It can be seen that the TANG generates energies up to $1 \mathrm{GHz}$. 


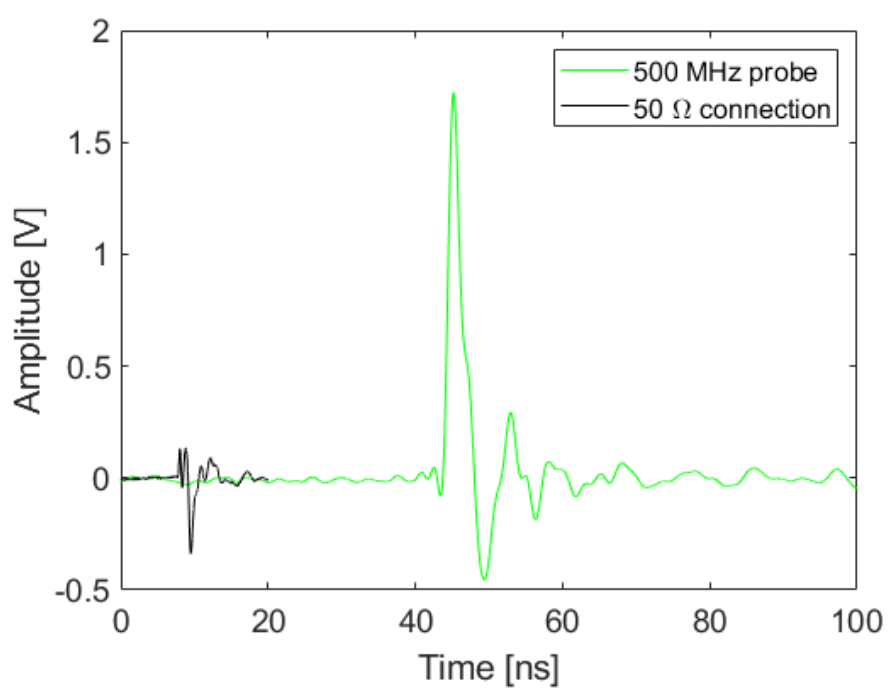

Fig. 4. Pulses sampled with two different probes. Here the individual $p(t)$ is shown.

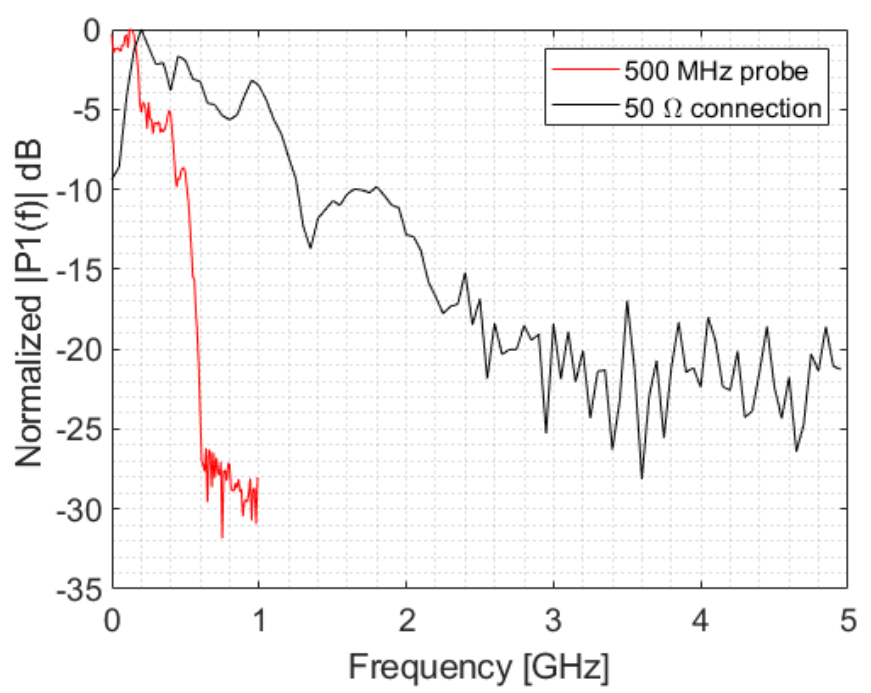

Fig. 5. Pulses sampled with two different probes - normalized power spectrum.

Individual pulses were periodically generated with a PRF (pulse repetition frequency) which is directly dependent on the DC voltage V1. It was found experimentally to be around $16 \mathrm{MHz}$ for DC supply voltages of $70 \mathrm{~V}$ for the TANG presented in Fig. 1. By increasing the DC voltage the time it takes for $C l$ to reach the avalanche voltage is smaller, so increasing the PRF.

\section{RADIATION MEASUREMENT}

The antenna was simulated with FEKO commercial package, based on the Method of Moments. Fig 6 shows the computed gain at the direction of its maximum value, in accordance with the inset of the figure. The computed input impedance response is shown in Fig. 7, in both real and imaginary parts. It can be seen that it has a strong capacitive part, due to the small ground plane. 


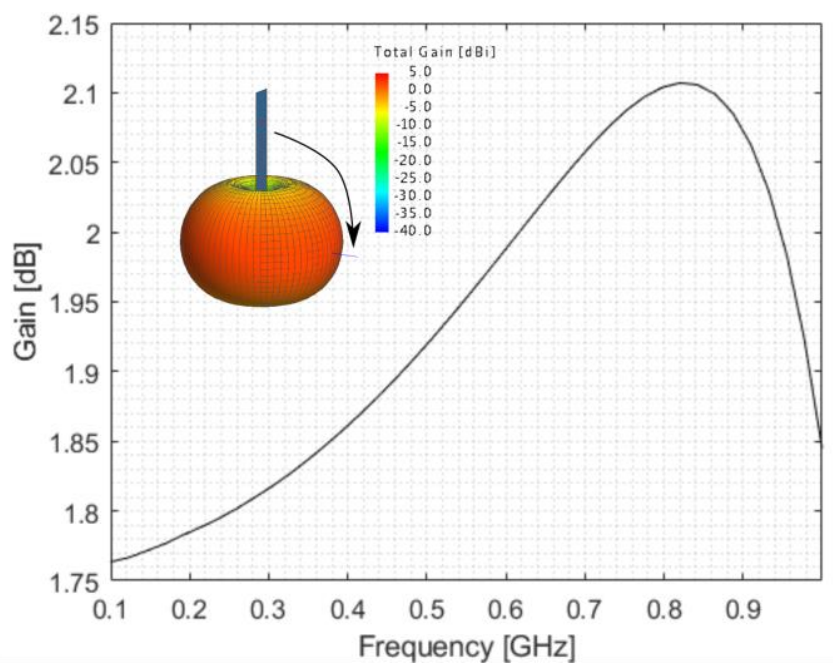

Fig. 6. Simulated gain according to the direction of the arrow on the inset. In detail the 3D gain plot at the frequency of 700 $\mathrm{MHz}$.

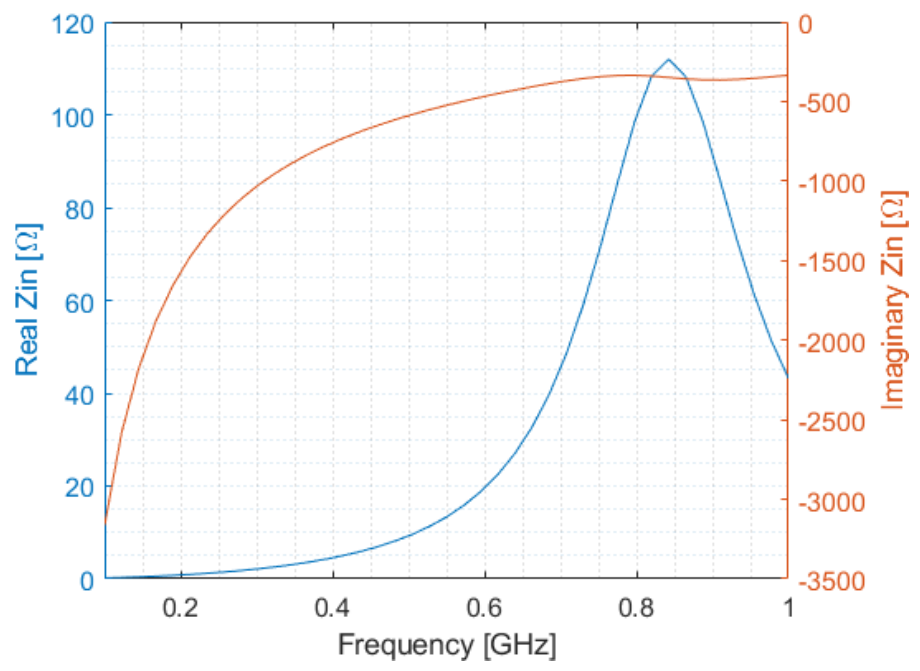

Fig. 7. Computed TANG antenna input impedance, real and imaginary parts.

The connection of the monopole to the collector resulted in pulses severely distorted and with low amplitudes, due to the higher impedance on that point - that is the reason the emitter connection was used instead, which presents a lower output impedance. Loop antennas, also experimentally investigated, placed in series with the emitter resulted in low performance concerning both radiation and the damping of the oscillations. Since the noise generated has a broad bandwidth shape, the antenna has to be simple, low profile (given the dimensions of the circuit alone) and with a large bandwidth as possible. Fig. 8 shows the comparison of the radiated energy, captured on a spectrum analyzer connected to a monopole antenna at 1 meter of the TANG. Two measurements sets were carried out with and without the antenna. The site was not electromagnetically shielded so noise from 
broadcast and mobile phone carriers were picked up during the measurement. It can be seen that specifically around $600 \mathrm{MHz}$ the antenna radiates the energy with maximum efficiency.

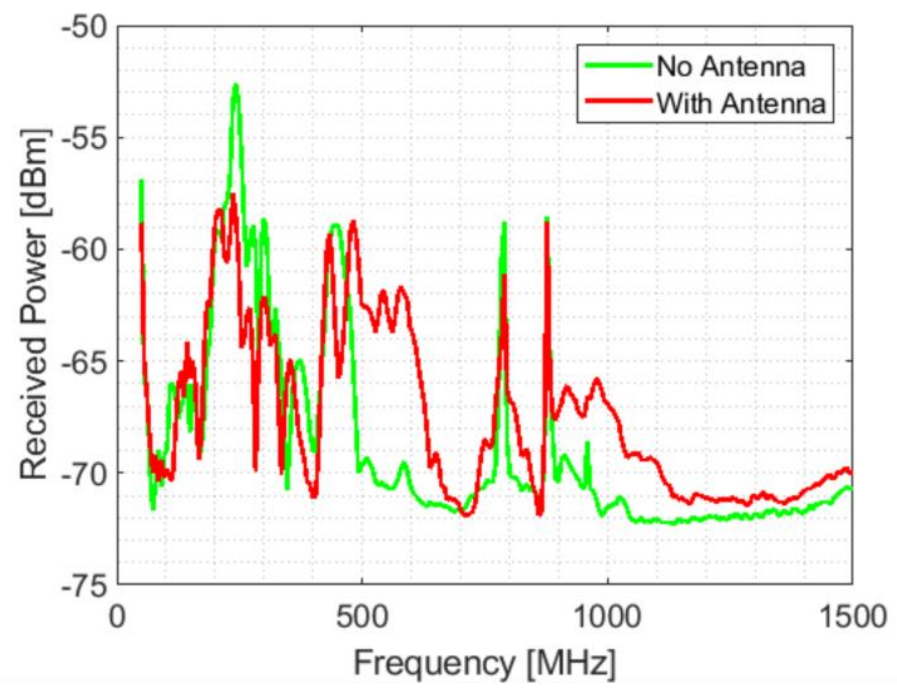

Fig. 8. Comparison of the radiated power with and without the antenna connected to the TANG.

For the sake of better shielding, the TANG was inserted into a shielded cavity. The oscillator energy was sampled inside the box by a monopole antenna connected to a receiver (Rohde \& Schwarz FSQ), as Fig. 9 shows. A wood box was internally covered with a $2 \mathrm{~mm}$-thick aluminum sheet, with the lid also covered with the same sheet. Proper galvanic contact between the lid and box sheet was ensured by means of screws. Cabling (DC supply and the receiver monopole) was placed inside the box through a hole on top of the box.

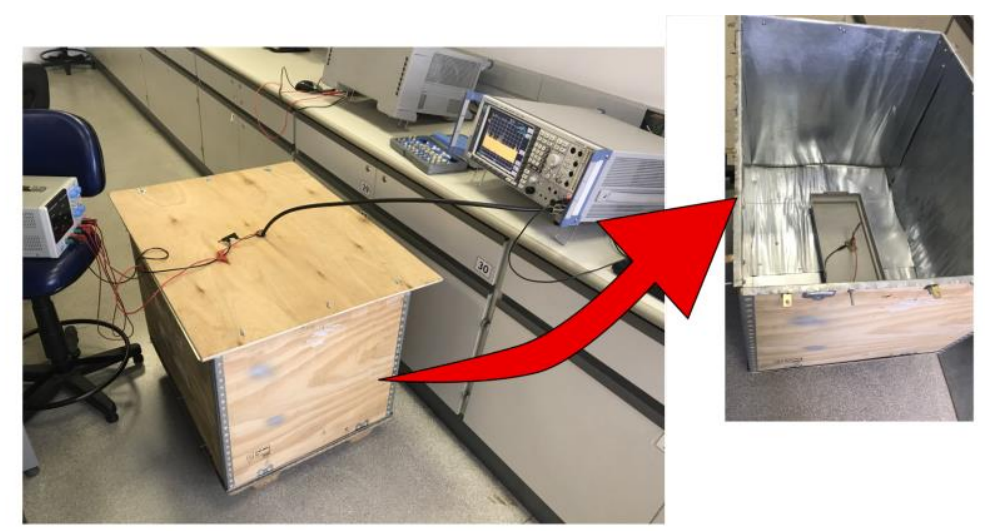

Fig. 9. Shielded box used to measure the radiated noise from the TANG. On the right, detail of the TANG isolated by the metallic ground by a plastic insulator.

The results for two different tests are shown in Fig. 10. Two measurements were performed, using different circuits but with the same component values. The setup used the "max hold" option to ensure 
that successive runs capture all emissions within the frequency range. Each run was set to last three minutes. The curve named noise floor shows the existing carriers (broadcast, TV, mobile phone, etc) without the TANG on. It can be seen that the TANG emissions run into the $1 \mathrm{GHz}$ range, in spite of the antenna made resonant to frequencies close to $600 \mathrm{MHz}$. Signals close to $600 \mathrm{MHz}$ reached power levels close to $-40 \mathrm{dBm}$.

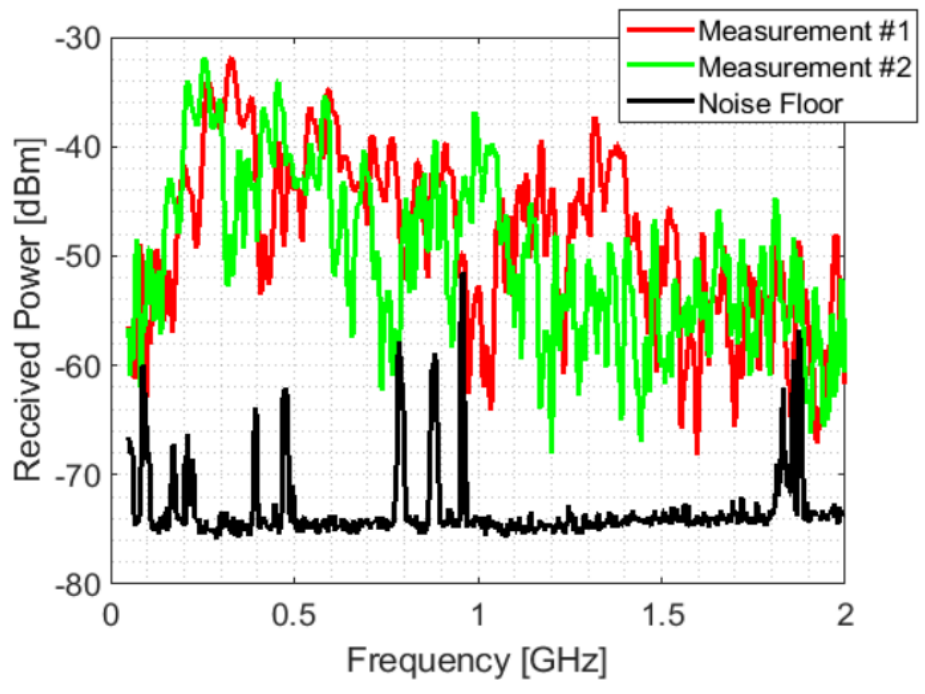

Fig. 10. Emissions of the TANG with two different sets.

Table I shows a comparison with similar designs. The computed maximum frequency is estimated from the rise or fall time, when not stated in the publication. It can be seen that the present design based on a single off-the-shelf transistor and operating with a lower amplitude bias voltage can achieve a high output frequency, in terms of radiated energy.

TABLE I. PERFORMANCE COMPARISON WITH SELECTED CIRCUITS PRESENTED IN THE LITERATURE

\begin{tabular}{|c|c|c|c|}
\hline Type & $\begin{array}{c}\text { Max. Freq. } \\
{[\mathbf{M H z}]}\end{array}$ & $\begin{array}{c}\text { DC Bias } \\
{[\mathbf{V}]}\end{array}$ & Antenna coupled? \\
\hline 4 stage 2N3055 series-connected [2]. & 100 & 1380 & No \\
\hline 10 stage series Marx generator[4]. & 175 & $204-284$ & No \\
\hline 22 diode connected transistors [5]. & 110 & 6000 & No \\
\hline Stepped recovery diode circuit [6]. & 700 & - & No \\
\hline Balanced output avalanche transistor [9]. & 380 & 330 & Yes, TEM Horn antenna \\
\hline 11 stacked NPN transistors [12]. & 350 & 2000 & No \\
\hline Avalanche 2N5551 transistor, simulation only & 180 & 165 & No \\
\hline [13]. & 1000 & 75 & Yes, planar monopole \\
\hline This work. & & & \\
\hline
\end{tabular}




\section{CONCLUSION}

The TANG circuit in spite of its simplicity generated electromagnetic noise with frequencies up to $1 \mathrm{GHz}$, with pulses in the sub-nanosecond range. A single off-the-shelf bipolar transistor operating in the avalanche mode and connected to a planar monopole antenna enabled the operation of the circuit as a stand-alone jammer unit. Measurements in both time and frequency domain showed the pulse generation and this subsequent radiation as an electromagnetic wave.

\section{REFERENCES}

[1] A. Ruengwaree, Design of UWB Radar Sensors. Kassel, Germany: Kassel University Press. 2008.

[2] A. M. Zou, H. B. Wang, Y. Z. Wang, and D. F. Cheng, "Nanosecond pulser based on serial connection of avalanche transistors," International Conference on Measuring Technology and Mechatronics Automation ICMTMA 2010, Changsa, China, 2010, vol. 2, no. 1, pp. 752-755.

[3] Q. Wu and W. Tian, "Design of electronic circuits of nanosecond impulser based on avalanche transistor," 11th Int. Conf. Electron. Packag. Technol. High Density Packag. ICEPT-HDP, Xi'an, China 2010, pp. 774-777.

[4] Q. Yang, X. Zhou, Q. G. Wang, and M. Zhao, "Comparative analysis on the fast rising edge pulse source with two kinds of avalanche transistor," 2013 Cross Strait Quad-Regional Radio Science and Wireless Technology Conference CSQRWC, Sichuan, China, 2013, pp. 447-450.

[5] Z. Zhong, Y. Liu, D. Zeng, and J. Tan, "Research on high-repetitionhigh-voltage nanosecond rectangular pulse generator based on avalanche transistor," 7th Asia-Pacific Conference on Environmental Electromagnetics CEEM , Hangzhou, China, 2015, pp. 73-76.

[6] P. Protiva, J. Mrkvica, and J. Machac, "Universal generator of ultrawideband pulse", Radioengineering, vol. 17, no. 4, pp. 74-78, Dec. 2008.

[7] S. A. M. K. A. Lukin, G. P. Pochanin, "Large-Current Radiator with Avalanche Transistor Switch," IEEE Trans. Electromagn. Compat., vol. 39, no. 2, pp. 156-160, May 1997.

[8] H. G. Schantz, "UWB Magnetic Antennas," IEEE Antennas and Propagation Society International Symposium Digest. Held in conjunction with: USNC/CNC/URSI North American Radio Sci. Meeting, Columbus, OH, USA, pp. 604-607, 2003.

[9] A. B. Ahmed Abbas H. Ameri, G"unter Kompa, "650 W Pulse Generator for Ultra-Wideband (UWB) Radar Application,” 6th German Microwave Conference, Darmstadt, Germany, pp. 198-201, 2011.

[10] V. Milovanovic and R. van der Toorn, "RF small signal avalanche characterization and repercussions on bipolar transistor circuit design," IEEE Eurocon 2009, St. Petersburg, Russia, pp. 230-233, 2009.

[11] A. S. Sedra and K. C. Smith, Microelectronic Circuits, 7th ed. New York City, US: Oxford University Press, 2015.

[12] R. J. Baker, "High voltage pulse generation using current mode second breakdown in a bipolar junction transistor," Rev. Sci. Instrum., vol. 62, no. 4, pp. 1031-1036, April 1991.

[13] Q. Yin, Z. Pan and Z. Zhang, "Design of a High-Performance Ultra-Wideband Monocycle Pulse Generator", Advances in Engineering, Proceedings of the 2018 International Conference on Mechanical, Electronic, Control and Automation Engineering (MECAE 2018), vol. 149, pp. 158-164, 2018. 